\title{
ERRATA
}

\section{Magnitude determination using duration of high frequency energy radiation and displacement amplitude: application to tsunami earthquakes}

\author{
Tatsuhiko Hara
}

Earth Planets Space, Vol. 59, pp. 561-565, 2007

Since some waveform data in the original data set for the 1992 Nicaragua earthquake were merged incorrectly (the waveform data with different location IDs were merged), I reanalyzed this event with the corrected data set. The duration of high frequency energy radiation and magnitude for this earthquake are now $113.3 \mathrm{~s}$ and 7.49, respectively, while 139.4 and 7.52 in Hara (Earth Planets Space, 59, 561-565, 2007). The figures and tables should be replaced as given below, while the discussions and conclusions are not subject to correction.

Figure 1(a) and the caption on p. 562 should read as follows:

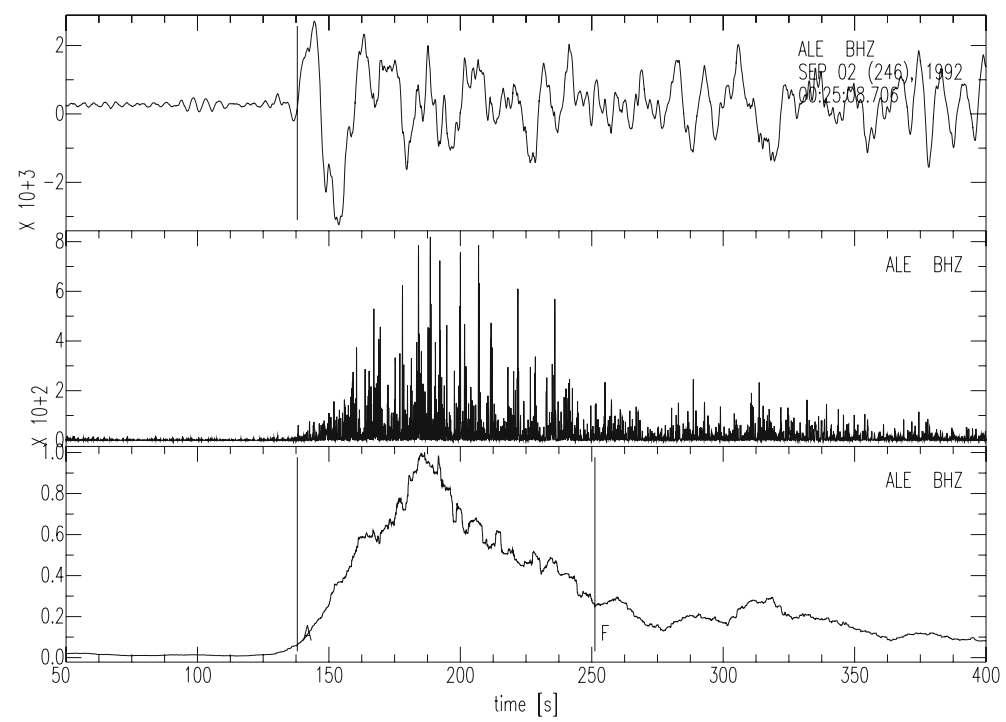

Fig. 1. Examples of measurements of high frequency energy radiation for the September 2, 1992 Nicaragua (a), the June 2, 1994 Java (b), the February 21, 1996 Peru (c), and the July 17, 2006 Java (d) earthquakes. The epicentral distances are 71.5, 46.4, 65.1 and 49.8 degrees, respectively. The top, middle and bottom traces in (a)-(d) are an observed seismogram, a time series of squares of band-pass (2-4 Hz) filtered seismogram, and its smoothed time series (normalized by the maximum value), respectively. "A" and "F" in the bottom traces denote arrivals of $P$-waves and estimated ends of high frequency energy radiation, respectively.

Tables 2 and 3 on p. 563 should read as follows:

Table 2. The measured durations of high frequency energy radiation and source time estimates.

\begin{tabular}{ccccc}
\hline Event & Duration $(\mathrm{s})$ & (C.T. - O.T. $) \times 2(\mathrm{~s})$ & Source time estimates (s) & No. of stations \\
\hline 1992 Nicaragua & 113.3 & 89 & $100-10^{(1)}$ & 16 \\
1994 Java & 96.5 & 78 & $80-90^{(2)}$ & 18 \\
1996 Peru & 78.1 & 45.4 & $50-60^{(3)}$ & 23 \\
2006 Java & 155.6 & 138.2 & $\geq 150^{(4)}$ & 26 \\
\hline
\end{tabular}

"Duration (s)" is the estimate of duration of high frequency energy radiation. "(C.T.-O.T.) $\times 2$ " is the doubled difference between centroid time in the Global CMT catalog and origin time in the USGS bulletin. "Source time estimates (s)" shows a source time estimate from previous studies. Their references are as follows. (1) Ide et al. (1993), Kanamori and Kikuchi (1993), Kikuchi and Kanamori (1995), Velasco et al. (1994), Ihmle (1996); (2) Abercrombie et al. (2001); (3) Ihmle et al. (1998), Bourgeois et al. (1999); (4) Ammon et al. (2006), Ji (2006), Yagi (2006). "No. of stations" is the number of stations used in this study. 
Table 3. The magnitude estimates obtained in this study and those of $M_{w p}, M_{w}\left(M_{m}\right)$, and $M_{w}$.

\begin{tabular}{ccccc}
\hline Event & $M$ (this study) & $M_{w p}$ & $M_{w}\left(M_{m}\right)$ & $M_{w}$ \\
\hline 1992 Nicaragua & 7.49 & $7.2^{(1)}$ & $7.55^{(2)}$ & 7.6 \\
1994 Java & 7.78 & $7.5^{(1)}$ & $7.65^{(2)}$ & 7.8 \\
1996 Peru & 7.35 & $7.5^{(1)}$ & $7.45^{(2)}$ & 7.5 \\
2006 Java & 7.66 & 7.2 & 7.4 & 7.7 \\
\hline
\end{tabular}

(1) Tsuboi (2000). (2) $M_{w}\left(M_{m}\right)$ was introduce by Weinstein and Okal (2005). We calculated this scale using the averages of the $M_{m}$ estimates presented by Newman and Okal (1998). $M_{w p}$ and $M_{w}\left(M_{m}\right)$ for the 2006 Java earthquake are from PTWC. $M_{w}$ is from the Global CMT catalog (although they are shown in Table 1, we show them here again for comparison).

Figure 2 on p. 563 should read as follows:

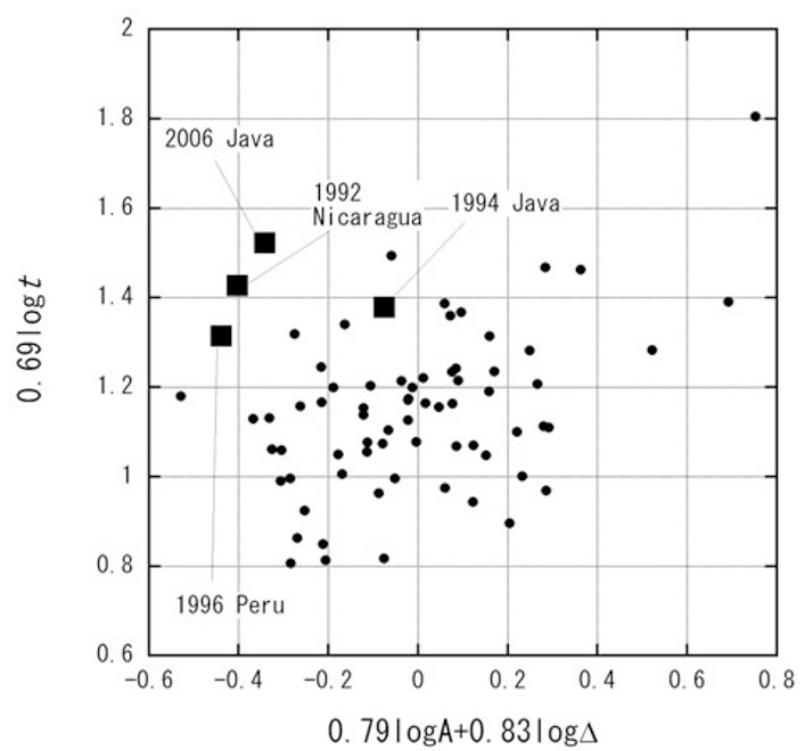

Fig. 2. Contributions to magnitudes from maximum displacement amplitudes and epicentral distances (the horizontal axis) and those from durations of high frequency energy radiation (the vertical axis) are plotted for the earthquakes analyzed in this study and those analyzed by Hara (2007) as squares and dots, respectively. 